$9-1-2014$

\title{
Adaptive Control of an Active Magnetic Bearing with External Disturbance
}

\author{
Lili Dong \\ Cleveland State University, I.dong34@csuohio.edu \\ Silu You \\ Cleveland State University
}

Follow this and additional works at: https://engagedscholarship.csuohio.edu/enece_facpub How does access to this work benefit you? Let us know!

\section{Publisher's Statement}

NOTICE: this is the author's version of a work that was accepted for publication in ISA

Transactions. Changes resulting from the publishing process, such as peer review, editing, corrections, structural formatting, and other quality control mechanisms may not be reflected in this document. Changes may have been made to this work since it was submitted for publication. A definitive version was subsequently published in ISA Transactions, 53, 5, (09-01-2014); 10.1016/j.isatra.2013.12.028

\section{Repository Citation}

Dong, Lili and You, Silu, "Adaptive Control of an Active Magnetic Bearing with External Disturbance" (2014). Electrical Engineering \& Computer Science Faculty Publications. 322.

https://engagedscholarship.csuohio.edu/enece_facpub/322

This Article is brought to you for free and open access by the Electrical Engineering \& Computer Science Department at EngagedScholarship@CSU. It has been accepted for inclusion in Electrical Engineering \& Computer Science Faculty Publications by an authorized administrator of EngagedScholarship@CSU. For more information, please contact library.es@csuohio.edu. 


\title{
Adaptive control of an active magnetic bearing with external disturbance
}

\author{
Lili Dong*, Silu You \\ Department of Electrical and Computer Engineering, Cleveland State University, Cleveland, OH 44115, USA
}

\section{Introduction}

A magnetic bearing is a bearing which supports a load (such as a rotor) using magnetic levitation [1]. Magnetic bearings are classified into two categories: passive and active ones. A passive magnetic bearing is composed of permanent magnets and its output flux cannot be controlled. An active magnetic bearing (AMB) is made of electromagnets and its output flux can be adjusted by changing the current on the coil. Therefore, AMB is more popular in practice than passive magnetic bearings due to its controllable output flux.

AMB has been broadly used in flywheel energy storage systems, turbo compressors, vacuum pumps, vehicle gyroscopes, and so on. AMB has several advantages over conventional ball or journal bearings. The most significant advantage is that since the AMB suspends rotor in a magnetic field, the rotor can spin at a high speed (up to $60,000 \mathrm{rpm}$ ) without contacting any mechanical part. The only friction in AMB is windage, which can be removed when $A M B$ is operated in vacuum enclosure. This frictionless feature also leads to low energy loss and the elimination of extra lubricating system [2]. In addition, AMB has a long life span due to its low equipment wear and its insensitivity to pressure and temperature changes. Nevertheless, an external disturbance can cause a large deviation of a rotor from its equilibrium position. Then the rotor would touch a stator, resulting in the failure of

\footnotetext{
* Corresponding author. Tel.: +1 216687 5312; fax: +1 2166875405. E-mail address: L.Dong34@csuohio.edu (L. Dong).
}

operation. Therefore the control of the rotor position becomes a crucial problem for $\mathrm{AMB}$ systems. A controller that is robust against external disturbance is ideal for the AMB.

Different control approaches have been reported for regulating the rotor position of AMB systems. PID control in [3-5] is a typical and efficient method to stabilize the rotor. A proportional gain controller is reported in [4]. The PID controller is simple to implement and easy to tune. However, it is not robust against disturbances and system uncertainties. Other than PID, Least Quadratic Regulator (LQR) control is designed and realized in a small size prototype AMB [6]. The performances of a PID controller, a cascaded PI/PD controller, and a LQR based control method are compared with each other in $[7,8]$ for AMB systems. It is discovered in $[7,8]$ that the LQR based controller has better performance than PID or PI/PD controller. However, the design and tuning of LQR based controller is time consuming and computationally complex [7]. Self sensing control of AMB is introduced in [9-12]. Self sensing refers to the controller design in the absence of an extra position sensor. The position information thus needs to be obtained by measuring the bearing coil current. A novel approach called Active Disturbance Rejection Control (ADRC) developed in recent years is simulated on a self sensing AMB system in $[2,12]$. The ADRC demonstrates an excellent disturbance rejection capability through combining a PD controller with a linear extended state observer [12]. However, there is a steady state error in the position response for the ADRC controlled AMB system [12].

In this paper, an adaptive back stepping control (ABC) method is originally applied to a linearized model of the AMB. The $A B C$ 
developed in recent decades [13-22] is an advanced control approach including recursive feedback control, Lyapunov stability and adaptive law. In $[19,20]$, the $A B C$ is also combined with neural and fuzzy integral action. It is shown in [18] that the $A B C$ is superior to PI/PD or PID controller in its robustness against system uncertainties. Therefore $A B C$ has been successfully applied to inverted pendulum, robot manipulator, jet engine, helicopter, and induction motor drive [13-21]. In this paper, a regular $A B C$ and a novel adaptive observer based back stepping controller $(\mathrm{AOBC})$ are developed on the AMB system respectively. While the regular $A B C$ is based on the feedback information of three states (displacement, velocity, and current) from $A M B$, the $A O B C$ is constructed on only one state (displacement). In [23], some preliminary results are reported about the application of a regular $A B C$ to the $A M B$. The $A O B C$ is an alternative solution to the control problem of the AMBs where current and velocity are not measurable. It is demonstrated in this paper that $A B C$ and $A O B C$ are robust against both external disturbance and parameter variations. But the $A B C$ in [13-21] only compensates the variations of system parameters. Moreover, in this paper, the $A B C$ is constructed based on position feedback. So a steady state error would be eliminated in the displacement of the rotor. The control systems' stability is verified by Lyapunov's direct method.

The rest of this paper is organized as follows. The dynamic modeling of the AMB system is given in Section 2. The design of $A B C$ is presented in Section 3. The stability and robustness analyses for $A B C$ are demonstrated in Section 3 as well. The development of AOBC is presented in Section 4. The simulation results are shown in Section 5. Concluding remarks and future research are given in Section 6.

\section{Dynamic modeling of an AMB system}

In a typical stable AMB model, the rotor is levitated at its equilibrium point which is positioned right in the middle of two magnets. The two opposite electro magnets are trying to pull the rotor on each side in the absence of any external force. When an external force causes a displacement of the rotor from its equilibrium position, the displacement will be sensed by a position sensor. Position sensor outputs the position information to an electronic control system, which increases the current in one direction and decreases the current in another direction through the respective electro magnets. This produces a differential force to push the rotor to its original position. The signal from the electronic controller continuously updates the differential force to stabilize the rotor till no position error (between rotor's position and equilibrium position) is sensed.

Fig. 1 shows a simple magnetic actuator model. In this figure, $I$ is the coil current, $g$ is air gap, $N$ is the number of coil rounds on the core, $A_{g}$ represents the cross section area and $g$ is the air gap, $l$ is the length of the path enclosing a surface through which the

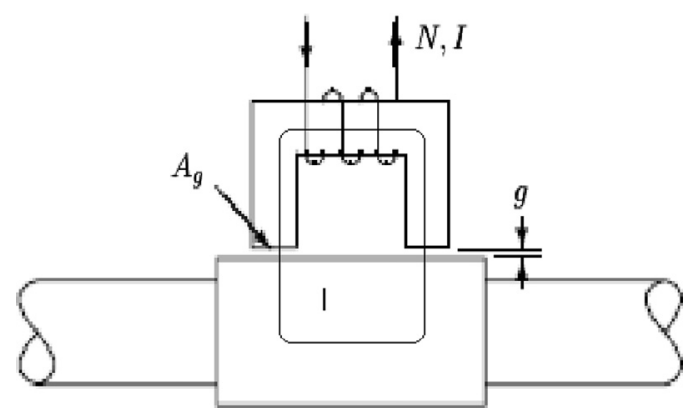

Fig. 1. Magnetic actuator. current flows. The magnetic field generated by the current will create an upward force.

According to Ampere's loop law, we have (1), where $H$ is the magnetic field, $n_{s}$ is the number of the segments through the path $l$, and $n_{c}$ is the number of different coils.

$\sum_{i=1}^{n_{s}} H_{i} l_{i}=\sum_{i=1}^{n_{c}} N_{i} I_{i}$

Assuming that the permeability of the mediums $\mu$ is constant in each segment, we will have the magnetic flux density $B_{i}$ given by

$B_{i}=\mu_{i} H_{i}$

Combining (1) and (2) yields

$\sum_{i=1}^{n_{s}} \frac{B_{i} l_{i}}{\mu_{i}}=\sum_{i=1}^{n_{c}} N_{i} I_{i}$

For the system in Fig. 1, there are two air gaps and the permeability of air $\left(\mu_{g}\right)$ is much less than that of iron $\left(\mu_{0}\right)$. Then from (3), we will have

$2 \frac{B g}{\mu_{g}}=N I \Rightarrow B=\frac{\mu_{g} N I}{2 g}$

The energy $E$ stored in the air gaps is represented by

$E=A_{g} g \int H d B=A_{g} g H B$

where $H$ is constant. The electromagnetic force $(F)$ is the derivative of the energy $E$ with respect to air gap. Considering (5) and (2), we can calculate the electromagnetic force $F$ as

$F=\frac{d E}{d g}=B H A_{g}=\frac{1}{\mu_{g}} B^{2} A_{g}$

With the equation of flux density in (4), we can rewrite (6) as

$F=\frac{\mu_{g} N^{2} I^{2} A_{g}}{4 g^{2}}$

In this paper, we use a one degree of freedom (DOF) AMB model as shown in Fig. 2.

In Fig. 2, $F_{d}$ is a disturbance force on the rotor, and $F_{1}$ and $F_{2}$ are two opposite electromagnetic forces, whose values are calculated through (7). The rotor in the middle of two cores is levitated and rotates in a plane perpendicular to the figure. We can adjust the input voltage $u_{1}$ and $u_{2}$ to control the two currents $i_{1}$ and $i_{2}$ so as to determine the resultant force. In Fig. 2, the displacement of rotor from nominal position $x_{0}$ is $x$, and $m$ is the rotor's mass. According to Newton's law, we have

$m \ddot{x}=F_{1}+F_{d}-F_{2}$

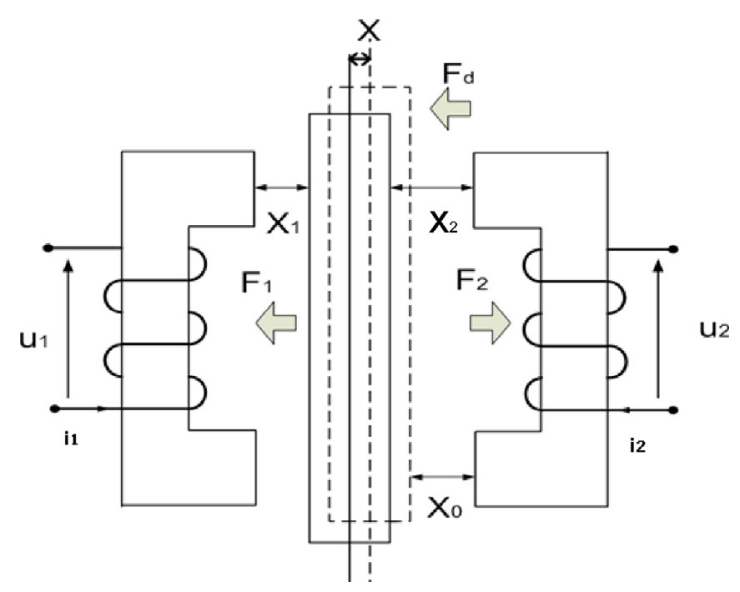

Fig. 2. AMB model. 
In Fig. 2, $x_{1}$ and $x_{2}$ are the air gaps between the rotor and left and right stators respectively. Replacing $g$ in (7) with $x_{1}$ and $x_{2}$ separately, we can derive the two electromagnetic forces $F_{1}$ and $F_{2}$ as follows:

$F_{1}=\frac{\mu_{g} N^{2} i_{1}^{2} A_{g}}{4 x_{1}^{2}}=\frac{K}{4}\left(\frac{i_{1}}{x_{1}}\right)^{2}, \quad F_{2}=\frac{\mu_{g} N^{2} i_{2}^{2} A_{g}}{4 x_{2}{ }^{2}}=\frac{K}{4}\left(\frac{i_{2}}{x_{2}}\right)^{2}$

where $K=\mu_{g} N^{2} A_{g}$. According to Kirchoff's Voltage Law (KVL), we have

$u_{1}=R i_{1}+L_{s} \frac{d i_{1}}{d t}+\frac{K}{2} \frac{d}{d t}\left(\frac{i_{1}}{x_{1}}\right), \quad u_{2}=R i_{2}+L_{s} \frac{d i_{2}}{d t}+\frac{K}{2} \frac{d}{d t}\left(\frac{i_{2}}{x_{2}}\right)$

where $R$ is coil resistance, $L_{S}$ is the self inductance of coil, and $(k / 2)(d / d t)\left(i_{1} / x_{1}\right)$ and $(k / 2)(d / d t)\left(i_{2} / x_{2}\right)$ represent the back electromotive force (EMF) generated by the air gap flux change.

We suppose $\left(x_{0}, i_{0}, u_{0}\right)$ represents equilibrium states. From Fig. 2, we have

$x_{1}=x_{0}-x, \quad x_{2}=x_{0}+x$

$i_{1}=i_{0}+i, \quad i_{2}=i_{0}-i$

$u_{1}=u_{0}+u, \quad u_{2}=u_{0}-u$

Substituting (11) through (13) into (10) and substituting (9) into (8), we will have a nonlinear system model given by

$\dot{x}=v$

$\dot{v}=\frac{K}{4 m}\left(\frac{i_{1}}{x_{0}-x}\right)^{2}-\frac{K}{4 m}\left(\frac{i_{2}}{x_{0}+x}\right)^{2}+\frac{F_{d}}{m}$

$\dot{i}_{1}=\frac{2\left(x_{0}-x\right)}{2 L_{s}\left(x_{0}-x\right)+K}\left[-R i_{1}-\frac{K}{2\left(x_{0}-x\right)^{2}} v i_{0}+u_{1}\right]$

$\dot{i}_{2}=\frac{2\left(x_{0}+x\right)}{2 L_{s}\left(x_{0}+x\right)+K}\left[-R i_{2}-\frac{K}{2\left(x_{0}+x\right)^{2}} v i_{1}+u_{2}\right]$

where $v$ is the velocity of rotor. We use Jacobian transformation to linearize the nonlinear model (14) around equilibrium states. The linearized state equations are given by (15) and (16). The state matrix is represented by $A$. Electromagnets are biased with a current $i_{0}$. According to [9], as the current $i_{0}$ is constant, the bias voltage caused by the coil resistance $R$ is $u_{0}=R i_{0}$. As the current $i_{0}$ is varying, the relationship between $i_{0}$ and $u_{0}$ is given by (16).

$\left[\begin{array}{c}\dot{x} \\ \dot{v} \\ \dot{i}\end{array}\right]=\underbrace{\left[\begin{array}{ccc}0 & 1 & 0 \\ \frac{2 k_{s}}{m} & 0 & \frac{2 k_{i}}{m} \\ 0 & \frac{-k_{i}}{L_{0}+L_{s}} & \frac{-R}{L_{0}+L_{s}}\end{array}\right]}_{A}\left[\begin{array}{c}x \\ v \\ i\end{array}\right]+\left[\begin{array}{c}0 \\ 0 \\ \frac{1}{L_{0}+L_{s}}\end{array}\right] u+\left[\begin{array}{c}0 \\ \frac{1}{m} \\ 0\end{array}\right] F_{d}$

$\frac{d}{d t} i_{0}=\frac{-R}{L_{0}+L_{s}} i_{0}+\frac{1}{L_{0}+L_{s}} u_{0}$

where

$k_{s}=\frac{K}{2} \frac{i_{0}^{2}}{x_{0}^{3}}, \quad k_{i}=\frac{K}{2} \frac{i_{0}}{x_{0}^{2}}$ and $L_{0}=\frac{K}{2 x_{0}}$.

The parameter values for the AMB system are given in Table 1.

From (15) and the parameter values listed in Table 1, we can calculate the eigenvalues of $A$, which are [202.5781, - 179.4896, $-70.1937]$. Since there is a positive eigenvalue for matrix $A$, the system is inherently unstable. An effective controller is crucial to stabilize the AMB. The controller is constructed on the linearized model represented by (15) and (16).

\section{ABC design and stability proof}

Since AMB is an unstable system, the primary control objectives are to stabilize the AMB and to drive the position of the rotor
Table 1

Parameter values.

\begin{tabular}{lll}
\hline Parameter & Symbol & Value (unit) \\
\hline Force-displacement constant & $K_{s}$ & $142,860 \mathrm{~N} / \mathrm{m}$ \\
Force-current constant & $K_{i}$ & $100 \mathrm{~N} / \mathrm{A}$ \\
Coil self inductance & $L_{s}$ & $120 \mathrm{mH}$ \\
Air gap inductance & $L_{0}$ & $70 \mathrm{mH}$ \\
Mass of rotor & $m$ & $4.6 \mathrm{~kg}$ \\
Coil resistance & $R$ & $8 \Omega$ \\
Nominal air gap & $x_{0}$ & $0.0007 \mathrm{~m}$ \\
Bias current & $i_{0}$ & $1 \mathrm{~A}$ \\
Disturbance force & $F_{d}$ & $4.6 \mathrm{~N}$ \\
\hline
\end{tabular}

to its equilibrium point in the presences of an external disturbance and system uncertainties. It is also desired that the disturbance be estimated accurately so it can be canceled in the control effort. It should be mentioned that a nonlinear $A B C$ has been applied to an AMB system in [21]. The nonlinear ABC treated the coil current as input and all the parameters that are associated with the position of the rotor are taken as unknown dynamics. The problem stated in [21] is different from the one in this paper because we take system's input as voltage and we only assume an external disturbance as an unknown parameter.

\subsection{Model transformation}

Adaptive back stepping controller consists of two parts: backstepping controller and adaptive laws. The back stepping controller is used to stabilize and control rotor's position. Adaptive law estimates the disturbance. The general control Lyapunov function (CLF) is constructed to include the rotor's displacement, the errors between real system states and their stabilizing functions, and the difference between estimated and real disturbance. In the design of $A B C$, the AMB system has to be expressed as a "strict feedback form" $[22,23]$. Eq. (15) can be rewritten as

$\left[\begin{array}{c}\dot{x} \\ \dot{v} \\ \dot{i}\end{array}\right]=\left[\begin{array}{lll}0 & 1 & 0 \\ a & 0 & b \\ 0 & c & d\end{array}\right]\left[\begin{array}{l}x \\ v \\ i\end{array}\right]+\left[\begin{array}{l}0 \\ 0 \\ e\end{array}\right] u+\left[\begin{array}{l}0 \\ f \\ 0\end{array}\right] F_{S}$

where

$a=\frac{2 k_{s}}{m}, \quad b=\frac{2 k_{i}}{m}, \quad c=\frac{-k_{i}}{L_{0}+L_{s}}, \quad d=\frac{-R}{L_{0}+L_{s}}, \quad e=\frac{1}{L_{0}+L_{s}}, \quad f=\frac{1}{m}$, and $F_{s}=F_{d}$

For creating a "strict feedback form", (17) can be transformed into (18), (19) and (20), where

$x_{1}=\frac{1}{b} x, \quad x_{2}=\frac{1}{b} v, \quad x_{3}=i$.

$\dot{x}_{1}=x_{2}$

$\dot{x}_{2}=x_{3}+\frac{a}{b} x_{1}+\theta=x_{3}+\varphi_{1}\left(\theta, x_{1}\right)$

$\dot{x}_{3}=u^{\prime}+c b x_{2}+d x_{3}=u^{\prime}+\varphi_{2}\left(x_{2}, x_{3}\right)$

In (19) and (20), the disturbance force and control effort are defined as $\theta=F_{s} /(b m)$, and $u^{\prime}=e u$ respectively. In addition, $\varphi_{1}\left(\theta, x_{1}\right)=(a / b) x_{1}+\theta$, and $\varphi_{2}\left(x_{2}, x_{3}\right)=c b x_{2}+d x_{3}$.

\subsection{Controller design and stability proof}

Our control goal is to regulate the position of the rotor $x_{1}$ in the presence of disturbance. In (18), we suppose that the virtual control $x_{2}$ can be used to drive $x_{1}$ to zero. Then we take $\alpha_{1}=-c_{1} x_{1}$, where $c_{1}$ is a positive real number, as stabilizing 
function or virtual controller (virtual state) to replace $x_{2}$. If $x_{2}=\alpha_{1}$, the desired state $x_{1}$ will be asymptotically stable by constructing CLF : $V=(1 / 2) x_{1}^{2}$ (where $\left.\dot{V}=-c_{1} x_{1}^{2}\right)$. However, since there is an error between $x_{2}$ and $\alpha_{1}$, we need to construct new state space equations called "error system" whose states are the differences between the real states and their stabilizing functions. The error states should be driven to zeros. The control goal then becomes asymptotically stabilizing all the states of the error system. We take the displacement $x_{1}$ as the first state $z_{1}$ of the error system, hence $z_{1}=x_{1}$. The error between second state $x_{2}$ and its stabilizing function $\alpha_{1}$ is $z_{2}=x_{2}-\alpha_{1}$. Then the CLF consisting of these two states is

$V_{1}=\frac{1}{2} z_{1}^{2}+\frac{1}{2} z_{2}^{2}$

Since (21) is the CLF for (18) and (19), our task is to find a suitable input denoted by the virtual control $x_{3}$ to make the derivative of (21) negative definite so that the two terms $z_{1}$ and $z_{2}$ will be driven to zero eventually. Even if the derivative is negative semi-definite, LaSalle-Yoshizawa theorem shows that $x_{1}$ will still be driven to zero. With (18) and (19), we can calculate the derivative of (21), which is

$\dot{V}_{1}=x_{1} x_{2}+z_{2}\left(x_{3}+\varphi_{1}-\frac{\partial \alpha_{1}}{\partial x_{1}} x_{2}\right)$

where $x_{2}=z_{2}+\alpha_{1}$. We choose $x_{3}$ as virtual control signal. If the second stabilization function is given by

$x_{3}=\alpha_{2}=-c_{2} z_{2}-\varphi_{1}+\frac{\partial \alpha_{1}}{\partial x_{1}} x_{2}-z_{1}$,

where $c_{2}$ is a positive real number, the derivative of $V_{1}$ will become $-c_{1} z_{1}^{2}-c_{2} z_{2}^{2}$ which is negative definite. However, there is still an error $z_{3}=x_{3}-\alpha_{2}$ being existent. So a new CLF including all the existing errors and displacement is created as

$V_{2}=\frac{1}{2} z_{1}^{2}+\frac{1}{2} z_{2}^{2}+\frac{1}{2} z_{3}^{2}$

The derivative of $V_{2}$ is

$\dot{V}_{2}=-c_{1} z_{1}^{2}-c_{2} z_{2}^{2}+z_{2} z_{3}+z_{3}\left(u^{\prime}+\varphi_{2}-\dot{\alpha}_{2}\right)$.

If $u^{\prime}$ is chosen as

$u^{\prime}=-c_{3} z_{3}-z_{2}-\varphi_{2}+\dot{\alpha}_{2}$,

where $c_{3}$ is a positive real number, the derivative of $V_{2}$ will be

$\dot{V}_{2}=-c_{1} z_{1}^{2}-c_{2} z_{2}^{2}-c_{3} z_{3}^{2}$

which means the derivative of the final CLF is negative definite. So the control goal is achieved. The above procedure is under the consumption that no external disturbance exists. If there is one, we will have to generate an adaptive law to estimate the disturbance so as to compensate it. The estimated disturbance will be functioning as additional feedback information in control law. The details about disturbance estimation are given as follows.

Let disturbance be $\theta$, and the first estimated disturbance be $\hat{\theta}_{1}$. We have an estimation error $\tilde{\theta}_{1}=\theta-\hat{\theta}_{1}$. We add the quadratic form of $\tilde{\theta}_{1}$ to (21) and then form a new CLF (28). Positive real numbers $\gamma_{i}(i=1,2,3)$ are chosen as adaptive coefficients.

$V_{1}^{\prime}=\frac{1}{2} z_{1}^{2}+\frac{1}{2} z_{2}^{2}+\frac{1}{2 \gamma_{1}} \tilde{\theta}_{1}^{2}$

Note that the disturbance $\theta$ is constant. Then the derivative of $V_{1}^{\prime}$ becomes

$\dot{V}_{1}^{\prime}=z_{1} z_{2}-c_{1} z_{1}^{2}+z_{2}\left(z_{3}+\alpha_{2}+\frac{a}{b} x_{1}+\theta-\dot{\alpha}_{1}\right)-\frac{1}{\gamma} \tilde{\theta}_{1} \dot{\hat{\theta}}_{1}$
We reselect $\alpha_{2}$ as

$\alpha_{2}=-z_{1}-c_{2} z_{2}-\frac{a}{b} x_{1}-\hat{\theta}_{1}+\dot{\alpha}_{1}$

Substituting (30) into (29) yields

$\dot{V}_{1}^{\prime}=-c_{1} z_{1}^{2}-c_{2} z_{2}^{2}+z_{2} z_{3}+\tilde{\theta}_{1}\left(z_{2}-\frac{1}{\gamma_{1}} \dot{\hat{\theta}}_{1}\right)$

In (31), we choose adaptive law as

$\dot{\hat{\theta}}_{1}=\gamma_{1} z_{2}$

Then the adaptive law in (32) will make the $\dot{V}_{1}^{\prime}$ negative definite assuming the term $z_{2} z_{3}$ in (31) could be canceled in the control effort later.

Next we would include the quadratic form of $z_{3}$ into CLF, where $z_{3}$ is the difference between $x_{3}$ and the updated $\alpha_{2}$ in (30). When we calculate the derivative of the new CLF (including $z_{3}^{2}$ ), we need to use the derivative of updated $\alpha_{2}$ which becomes

$$
\begin{aligned}
\dot{\alpha}_{2} & =\frac{\partial \alpha_{2}}{\partial z_{1}} \dot{z}_{1}+\frac{\partial \alpha_{2}}{\partial x_{2}} \dot{x}_{2}+\frac{\partial \alpha_{2}}{\partial \hat{\theta}_{1}} \dot{\hat{\theta}}_{1} \\
& =\frac{\partial \alpha_{2}}{\partial z_{1}} \dot{z}_{1}+\frac{\partial \alpha_{2}}{\partial x_{2}}\left(x_{3}+\frac{a_{1}}{b} x_{1}+\theta\right)+\frac{\partial \alpha_{2}}{\partial \hat{\theta}_{1}} \dot{\hat{\theta}}_{1}
\end{aligned}
$$

In (33), the disturbance $\theta$ has to be replaced by an estimate of it. We suppose $\hat{\theta}_{2}$ is the second estimate of $\theta$, and the estimation error is $\tilde{\theta}_{2}=\theta-\hat{\theta}_{2}$. Then the complete CLF including disturbance estimation errors can be constructed as

$V_{2}^{\prime}=\frac{1}{2} z_{1}^{2}+\frac{1}{2} z_{2}^{2}+\frac{1}{2} z_{3}^{2}+\frac{1}{2 \gamma_{1}} \tilde{\theta}_{1}^{2}+\frac{1}{2 \gamma_{2}} \tilde{\theta}_{2}^{2}$

The control law that was derived before is repeated as follows:

$u^{\prime}=-c_{3} z_{3}-z_{2}-\varphi_{2}+\dot{\alpha}_{2}$

According to (33), we can rewrite (35) as

$u^{\prime}=-c_{3} z_{3}-z_{2}-\varphi_{2}+\frac{\partial \alpha_{2}}{\partial z_{1}} \dot{z}_{1}+\frac{\partial \alpha_{2}}{\partial x_{2}}\left(x_{3}+\frac{a}{b} x_{1}+\hat{\theta}_{2}\right)+\frac{\partial \alpha_{2}}{\partial \hat{\theta}_{1}} \dot{\hat{\theta}}_{1}$

Next we differentiate the CLF $\left(V_{2}^{\prime}\right)$ in (34) just as we did for the CLF in (24). Given the control law in (36), the derivative of $V_{2}^{\prime}$ becomes

$\dot{V}_{2}^{\prime}=-c_{1} z_{1}^{2}-c_{2} z_{2}^{2}-c_{3} z_{3}^{2}-\frac{1}{\gamma_{2}} \tilde{\theta}_{2} \dot{\hat{\theta}}_{2}-z_{3} \frac{\partial \alpha_{2}}{\partial x_{2}} \tilde{\theta}_{2}$

In order to make (37) negative definite, we need to eliminate the error parts which contain $\tilde{\theta}_{2}$. If we choose adaptive law as

$\dot{\hat{\theta}}_{2}=-z_{3} \gamma_{2} \frac{\partial \alpha_{2}}{\partial x_{2}}$,

the derivative of $V_{2}^{\prime}$ will become

$\dot{V}_{2}^{\prime}=-c_{1} z_{1}^{2}-c_{2} z_{2}^{2}-c_{3} z_{3}^{2}$

Now that the derivative of the final CLF is negative definite, the AMB system is successfully stabilized at its equilibrium point. The control law expressed in (36) and the adaptive laws represented by (32) and (38) constitute the ABC for the AMB system with an external disturbance.

\subsection{Closed-loop control system}

The error system's state equations are represented by (40), where $Z$ is error state vector, vector $\tilde{\theta}$ consists of the estimation errors of external disturbance, and vector $\hat{\theta}$ includes estimated disturbances. The definitions of matrices $C, D$, and $E$ are indicated in (40). Based on (40), we can use Fig. 3, the closed-loop adaptive system for $A B C$, to generate adaptive laws. In Fig. 3, we define $\theta_{\text {vector }}=\left[\begin{array}{ll}\theta_{1} & \theta_{2}\end{array}\right]^{T}$. Fig. 4 shows the closed-loop diagram of $\mathrm{ABC}$ controlled AMB system. In Fig. 4, the reference signal $r$ is zero. Three system states $(x, v$, and $i)$ of an AMB plant are used to 


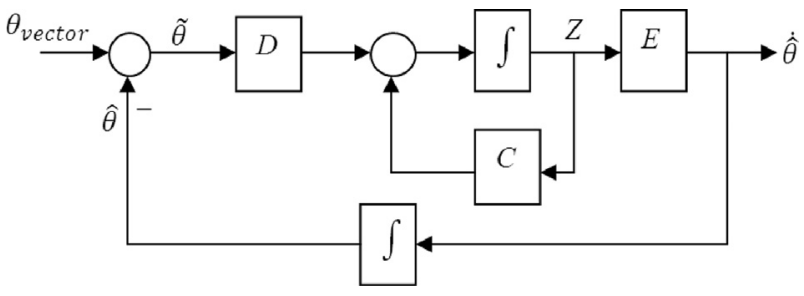

Fig. 3. Block diagram of the adaptive system for $A B C$.

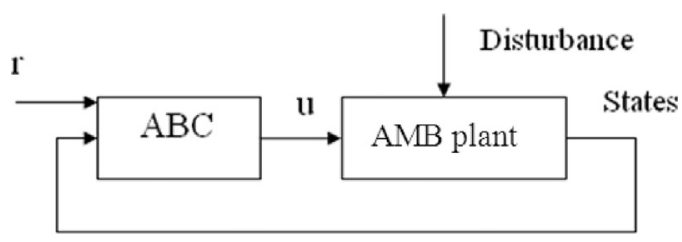

Fig. 4. Block diagram of $A B C$ controlled $A M B$ system.

construct $\mathrm{ABC}$. An external disturbance is applied to the AMB system.

$$
\begin{aligned}
& \underbrace{\left[\begin{array}{c}
\dot{z}_{1} \\
\dot{z}_{2} \\
\dot{z}_{3}
\end{array}\right]}_{\dot{Z}}=\underbrace{\left[\begin{array}{lll}
-c_{1} & 1 & 0 \\
-1 & -c_{2} & 1 \\
0 & -1 & -c_{3}
\end{array}\right]}_{\dot{C}} \underbrace{\left[\begin{array}{c}
z_{1} \\
z_{2} \\
z_{3}
\end{array}\right]}_{Z} \underbrace{\left[\begin{array}{ll}
0 & 0 \\
1 & 0 \\
0 & \frac{\partial \alpha_{2}}{\partial x_{2}}
\end{array}\right]}_{\dot{D}} \underbrace{\left[\begin{array}{c}
\tilde{\theta}_{1} \\
\tilde{\theta}_{2}
\end{array}\right]}_{\tilde{\theta}} \\
& \underbrace{\left[\begin{array}{c}
\dot{\hat{\theta}}_{1} \\
\dot{\hat{\theta}}_{2}
\end{array}\right]}_{\dot{\hat{\theta}}}=\underbrace{\left[\begin{array}{lll}
0 & \gamma_{1} & 0 \\
0 & 0 & \frac{\partial \alpha_{2}}{\partial x_{2}}
\end{array}\right]}_{\tilde{E}}\left[\begin{array}{c}
z_{1} \\
z_{2} \\
z_{3}
\end{array}\right] .
\end{aligned}
$$

\section{AOBC design and stability proof}

The $A B C$ developed in Section 3 is based on the assumption that full state feedback is available. However, in reality, only position output of the AMB is generally measurable. In this section, we aim to develop an adaptive back-stepping controller with position feedback only. An observer is designed to observe the states of current and velocity. The $\mathrm{ABC}$ based on observed states are AOBC. Again, we use Lyapunov's direct method to develop the controller and adaptive laws.

\subsection{Model transformation}

In order to apply $A O B C$ to the $A M B$ system, we need to transform the system model to an observable form. Conducting Laplace transform on (15), we have

$$
\begin{aligned}
X(s)= & \frac{2 k_{i}}{m L s^{3}+m R s^{2}+2\left(k_{i}^{2}-L k_{s}\right) s-2 k_{s} R} U(s) \\
& +\frac{L s+R}{m L s^{3}+m R s^{2}+2\left(k_{i}^{2}-L k_{s}\right) s-2 k_{s} R} F_{d}(s)
\end{aligned}
$$

where $L=L_{0}+L_{s}$. The observable canonical form of (41) is

$$
\begin{aligned}
& {\left[\begin{array}{c}
\dot{x}_{1} \\
\dot{x}_{2} \\
\dot{x}_{3}
\end{array}\right]=\left[\begin{array}{ccc}
-\frac{R}{L} & 1 & 0 \\
\frac{-2\left(k_{i}^{2}-L k_{s}\right)}{m L} & 0 & 1 \\
\frac{2 k_{s} R}{m L} & 0 & 0
\end{array}\right]+\left[\begin{array}{c}
0 \\
0 \\
\frac{2 k_{i}}{m L}
\end{array}\right] u+\left[\begin{array}{c}
0 \\
\frac{1}{m} \\
\frac{R}{m L}
\end{array}\right] F_{d}} \\
& y=x_{1}
\end{aligned}
$$

In (42), the state variable $x_{1}$ is displacement, and is also the system's output $y$. However, due to the canonical from realization, the other two states $x_{2}$ and $x_{3}$ in (42) are not velocity and current any more. Instead, they do not have physical meanings but are just used to construct the state equations.

Define

$\psi_{1}(y)=-\frac{R}{L} \chi_{1}, \quad a^{\prime}=\frac{R}{L}, \psi_{2}(y)=\frac{-2\left(k_{i}^{2}-L k_{s}\right)}{m L} \chi_{1}$,

$b^{\prime}=\frac{-2\left(k_{i}^{2}-L k_{s}\right)}{m L}, \quad \psi_{3}(y)=\frac{2 k_{s} R}{m L} x_{1}, \quad c^{\prime}=\frac{2 k_{s} R}{m L}$,

$u^{\prime \prime}=\frac{2 k_{i}}{m L} u, \quad \vartheta=\frac{F_{d}}{m}$

The parameters $a^{\prime}, \quad b^{\prime}$ and $c^{\prime}$ are used for testing the robustness of the AOBC in the following section. Then the state equations in (42) can be expanded as

$\dot{x}_{1}=x_{2}+\psi_{1}(y)$

$\dot{x}_{2}=x_{3}+\psi_{2}(y)+\vartheta$

$\dot{x}_{3}=\psi_{3}(y)+u^{\prime \prime}+a^{\prime} \vartheta$.

\subsection{Observer design}

The state observer can be constructed as

$X=\xi_{0}+\vartheta \xi_{1}+\varepsilon$

where $X=\left[\begin{array}{lll}x_{1}, & x_{2}, & x_{3}\end{array}\right]^{T}$ is observed state vector, $\xi_{0}=$ $\left[\begin{array}{lll}\xi_{01} & \xi_{02} & \xi_{03}\end{array}\right]^{T}$ and $\xi_{1}=\left[\begin{array}{lll}\xi_{11} & \xi_{12} & \xi_{13}\end{array}\right]^{T}$ are filter vectors, and $\varepsilon=\left[\begin{array}{lll}\varepsilon_{1} & \varepsilon_{2} & \varepsilon_{3}\end{array}\right]^{T}$ is the vector of estimation errors. We suppose $k_{i}$ $(i=1,2,3)$ are real numbers. The elements of vectors $\xi_{0}$ and $\xi_{1}$ are defined as follows:

$\left\{\begin{array}{l}\dot{\xi}_{01}=k_{1}\left(y-\xi_{01}\right)+\xi_{02}+\psi_{1}(y) \\ \dot{\xi}_{02}=k_{2}\left(y-\xi_{01}\right)+\xi_{03}+\psi_{2}(y) \\ \dot{\xi}_{03}=k_{3}\left(y-\xi_{01}\right)+u^{\prime \prime}+\psi_{3}(y)\end{array}\right.$

$\left\{\begin{array}{c}\dot{\xi}_{11}=-k_{1} \xi_{11}+\xi_{12} \\ \dot{\xi}_{12}=-k_{2} \xi_{12}+\xi_{13}+1 \\ \dot{\xi}_{13}=-k_{3} \xi_{13}+a^{\prime}\end{array}\right.$

Next we will discuss how we choose the constants $k_{i}$ and why the observer represented by (44), (45) and (46) can successfully observe the state vector $X=\left[\begin{array}{lll}x_{1}, & x_{2}, & x_{3}\end{array}\right]^{T}$. We define a matrix

$A_{0}=\left[\begin{array}{lll}-k_{1} & 1 & 0 \\ -k_{2} & 0 & 1 \\ -k_{3} & 0 & 0\end{array}\right]$

and select the gain vector $K=\left[\begin{array}{lll}k_{1} & k_{2} & k_{3}\end{array}\right]^{T}$ to make $A_{0}$ Hurwitz. Then the system model (43) can be rewritten as

$\dot{X}=A_{0} X+K y+\left[\begin{array}{l}\psi_{1}(y) \\ \psi_{2}(y) \\ \psi_{3}(y)\end{array}\right]+\left[\begin{array}{c}0 \\ 1 \\ a^{\prime}\end{array}\right] \vartheta+\left[\begin{array}{l}0 \\ 0 \\ 1\end{array}\right] u^{\prime \prime}$

The two filters given by (45) and (46) can be rewritten as

$\dot{\xi}_{0}=A_{0} \xi_{0}+K y+\left[\begin{array}{l}\psi_{1}(y) \\ \psi_{2}(y) \\ \psi_{3}(y)\end{array}\right]+\left[\begin{array}{l}0 \\ 0 \\ 1\end{array}\right] u^{\prime \prime}$

$\dot{\xi}_{1}=A_{0} \xi_{1}+\left[\begin{array}{lll}0 & 1 & a^{\prime}\end{array}\right]^{T}$

Given (47)-(49), we can obtain the derivative of observation error as

$\dot{\varepsilon}=\dot{X}-\dot{\xi}_{0}-\vartheta \dot{\xi}_{1}=A_{0}\left(X-\xi_{0}-\vartheta \xi_{1}\right)=A_{0} \varepsilon$

From (50), since $A_{0}$ is a Hurwitz matrix, the estimation error vector $\varepsilon$ will exponentially converge to zero. 


\subsection{Controller design and stability proof}

In this section, a CLF needs to be constructed to include state estimation errors, virtual control errors, disturbance estimation errors, and the tracking error of the displacement of rotor. All of the errors have to be controlled to zeros. The adaptive and control laws are developed in a way that is similar to the one in Section 3. The first state $z_{1}^{\prime}$ of the error system is chosen as

$z_{1}^{\prime}=y$

The derivative of $z_{1}^{\prime}$ is

$\dot{z}_{1}^{\prime}=x_{2}+\psi_{1}(y)$

Since $x_{2}$ is non-measurable, it can be replaced by its observed state $\xi_{02}+\vartheta \xi_{12}+\varepsilon_{2}$ as given by (44). Then (52) becomes

$\dot{z}_{1}^{\prime}=\xi_{02}+\vartheta \xi_{12}+\varepsilon_{2}+\psi_{1}(y)$

In (53), the disturbance $\vartheta$ needs to be replaced by its estimate $\hat{\vartheta}_{1}$. So the estimation error for disturbance is $\tilde{\vartheta}_{1}=\vartheta-\hat{\vartheta}_{1}$. The first CLF $f_{1}$ is selected as

$f_{1}=\frac{1}{2}\left(z_{1}^{\prime}\right)^{2}+\frac{1}{d_{1}} \varepsilon^{T} P_{0} \varepsilon+\frac{1}{2 \gamma_{1}^{\prime}} \tilde{\vartheta}_{1}^{2}$

where $\gamma_{1}^{\prime}$ and $d_{1}$ are positive real numbers, $P_{0}$ is a positive definite and symmetric matrix and $A_{0}^{T} P_{0}+P_{0} A_{0}=-Q$, where $Q$ is an identity matrix. In (53), if we choose $\xi_{02}$ as a virtual controller and $\xi_{02}=\beta_{1}$, we will have

$\beta_{1}=-c_{1}^{\prime} z_{1}^{\prime}-d_{1} z_{1}^{\prime}-\hat{\vartheta}_{1} \xi_{12}-\psi_{1}(y)$

where $c_{1}^{\prime}>0, c_{1}^{\prime} \in R$. Replacing the $\xi_{02}$ in (53) with (55) yields

$\dot{z}_{1}^{\prime}=-c_{1}^{\prime} z_{1}^{\prime}-d_{1} z_{1}^{\prime}+\tilde{\vartheta}_{1} \xi_{12}+\varepsilon_{2}$

From (54), the derivative of $f_{1}$ is

$\dot{f}_{1}=z_{1}^{\prime} \dot{z}_{1}^{\prime}+\frac{1}{d_{1}}\left(\dot{\varepsilon}^{T} P_{0} \varepsilon+\varepsilon^{T} P_{0} \dot{\varepsilon}\right)+\frac{1}{\gamma_{1}^{\prime}} \tilde{\vartheta}_{1} \dot{\tilde{\vartheta}}_{1}$

Substituting (50) $\left(\dot{\varepsilon}=A_{0} \varepsilon\right.$ ) and (56) into (57) produces

$$
\begin{aligned}
\dot{f}_{1}= & -c_{1}^{\prime}\left(z_{1}^{\prime}\right)^{2}-\left(\sqrt{d_{1}} z_{1}^{\prime}-\frac{1}{2 \sqrt{d_{1}}} \varepsilon_{2}\right)^{2}+\tilde{\vartheta}_{1}\left(z_{1}^{\prime} \xi_{12}-\frac{1}{\gamma_{1}^{\prime}} \dot{\hat{\vartheta}}_{1}\right) \\
& +\frac{1}{4 d_{1}} \varepsilon_{2}^{2}-\frac{1}{d_{1}} \varepsilon^{T} \varepsilon
\end{aligned}
$$

where

$\frac{1}{4 d_{1}} \varepsilon_{2}^{2}-\frac{1}{d_{1}} \varepsilon^{T} \varepsilon=-\frac{1}{d_{1}} \varepsilon_{1}^{2}-\frac{3}{4 d_{1}} \varepsilon_{2}^{2}-\frac{1}{d_{1}} \varepsilon_{3}^{2}$.

From (58), we can see that if the adaptive law is chosen as

$\dot{\hat{\vartheta}}_{1}=\gamma_{1}^{\prime} z_{1}^{\prime} \xi_{12}$

Eq. (58) will be negative semi definite. We define the error between $\xi_{02}$ and $\beta_{1}$ as $z_{2}^{\prime}$, which is the second state of the error system. Then $z_{2}^{\prime}=\xi_{02}-\beta_{1}$. The derivative of $z_{2}^{\prime}$ is

$$
\begin{aligned}
\dot{z}_{2}^{\prime}= & \dot{\xi}_{02}-\dot{\beta}_{1}=\xi_{03}+\psi_{2}(y)+k_{2}\left(y-\xi_{01}\right)-\frac{\partial \beta_{1}}{\partial z_{1}^{\prime}}\left(\xi_{02}+\xi_{12} \vartheta+\varepsilon_{2}\right. \\
& \left.+\psi_{1}(y)\right)-\frac{\partial \beta_{1}}{\partial \hat{\vartheta}_{1}} \dot{\hat{\vartheta}}_{1}-\frac{\partial \beta_{1}}{\partial \xi_{12}} \dot{\xi}_{12}
\end{aligned}
$$

In (60), the disturbance $\vartheta$ has to be replaced by its second estimate $\hat{\vartheta}_{2}$. We define the estimation error as $\tilde{\vartheta}_{2}=\vartheta-\hat{\vartheta}_{2}$. Then the second CLF is selected as

$f_{2}=f_{1}+\frac{1}{2}\left(z_{2}^{\prime}\right)^{2}+\frac{1}{d_{2}} \varepsilon^{T} P_{0} \varepsilon+\frac{1}{2 \gamma_{2}^{\prime}} \tilde{\vartheta}_{2}^{2}$ where $d_{2}$ and $\gamma_{2}^{\prime}$ are positive real numbers. If we choose $\xi_{03}$ as the second virtual controller, and $\xi_{03}=\beta_{2}$, we will have

$$
\begin{aligned}
\beta_{2}= & -c_{2}^{\prime} z_{2}^{\prime}-d_{2}\left(\frac{\partial \beta_{1}}{\partial z_{1}^{\prime}}\right)^{2} z_{2}^{\prime}-\psi_{2}(y)-k_{2}\left(y-\xi_{01}\right) \\
& +\frac{\partial \beta_{1}}{\partial z_{1}^{\prime}}\left(\xi_{02}+\xi_{12} \hat{\vartheta}_{2}+\psi_{1}(y)\right)+\frac{\partial \beta_{1}}{\partial \hat{\vartheta}_{1}} \dot{\hat{\vartheta}}_{1}+\frac{\partial \beta_{1}}{\partial \xi_{12}} \dot{\xi}_{12}
\end{aligned}
$$

Replacing $\xi_{03}$ in (60) with (62) yields

$\dot{z}_{2}^{\prime}=-c_{2}^{\prime} z_{2}^{\prime}-d_{2}\left(\frac{\partial \beta_{1}}{\partial z_{1}^{\prime}}\right)^{2} z_{2}^{\prime}-\frac{\partial \beta_{1}}{\partial z_{1}^{\prime}} \xi_{12} \tilde{\vartheta}_{2}-\frac{\partial \beta_{1}}{\partial z_{1}^{\prime}} \varepsilon_{2}$

where $c_{2}^{\prime}>0, c_{2}^{\prime} \in R$. Substituting (63) into the derivative of $f_{2}$ produces

$$
\begin{aligned}
\dot{f}_{2}= & \dot{f}_{1}-c_{2}^{\prime}\left(z_{2}^{\prime}\right)^{2}-\left(\sqrt{d_{2}} \frac{\partial \beta_{1}}{\partial z_{1}^{\prime}} z_{2}^{\prime}+\frac{\varepsilon_{2}}{2 \sqrt{d_{2}}}\right)^{2}+\frac{1}{4 d_{2}} \varepsilon_{2}^{2}-\frac{1}{d_{2}} \varepsilon^{T} \varepsilon \\
& -\tilde{\vartheta}_{2}\left(\frac{\partial \beta_{1}}{\partial z_{1}^{\prime}} z_{2}^{\prime} \xi_{12}+\frac{1}{\gamma_{2}^{\prime}} \dot{\hat{\vartheta}}_{2}\right)
\end{aligned}
$$

where

$\frac{1}{4 d_{2}} \varepsilon_{2}^{2}-\frac{1}{d_{2}} \varepsilon^{T} \varepsilon=-\frac{1}{d_{2}} \varepsilon_{1}^{2}-\frac{3}{4 d_{2}} \varepsilon_{2}^{2}-\frac{1}{d_{2}} \varepsilon_{3}^{2}$.

From (58), we can see that if the second adaptive law is chosen as

$\dot{\hat{\vartheta}}_{2}=-\gamma_{2}^{\prime} \frac{\partial \beta_{1}}{\partial z_{1}^{\prime}} z_{2}^{\prime} \xi_{12}$

Eq. (64) will be negative semi definite. We define the error between $\xi_{03}$ and $\beta_{2}$ as $z_{3}^{\prime}$, which is the third state of the error system. Then $z_{3}^{\prime}=\xi_{03}-\beta_{2}$. The derivative of $z_{3}^{\prime}$ is

$\dot{z}_{3}^{\prime}=\psi_{3}(y)+u^{\prime \prime}+k_{3}\left(y-\xi_{01}\right)-\dot{\beta}_{2}$

In (66), the derivative of $\beta_{2}$ is

$$
\begin{aligned}
\dot{\beta}_{2}= & \frac{\partial \beta_{2}}{\partial z_{1}^{\prime}}\left(\xi_{02}+\xi_{12} \vartheta+\varepsilon_{2}+\psi_{1}(y)\right)+\frac{\partial \beta_{2}}{\partial z_{2}^{\prime}} \dot{z}_{2}^{\prime}+\frac{\partial \beta_{2}}{\partial \xi_{01}} \dot{\xi}_{01}+\frac{\partial \beta_{2}}{\partial \xi_{02}} \dot{\xi}_{02} \\
& +\frac{\partial \beta_{2}}{\partial \xi_{12}} \dot{\xi}_{12}+\frac{\partial \beta_{2}}{\partial \hat{\vartheta}_{1}} \dot{\hat{\vartheta}}_{1}+\frac{\partial \beta_{2}}{\partial \hat{\vartheta}_{2}} \dot{\hat{\vartheta}}_{2}
\end{aligned}
$$

The disturbance $\vartheta$ in (67) has to be replaced by its estimate $\hat{\vartheta}_{3}$. The estimation error is $\tilde{\vartheta}_{3}=\vartheta-\hat{\vartheta}_{3}$. If we choose $u^{\prime \prime}$ as

$$
\begin{aligned}
u^{\prime \prime}= & \frac{\partial \beta_{2}}{\partial z_{1}^{\prime}}\left(\xi_{02}+\xi_{12} \hat{\vartheta}_{3}+\psi_{1}(y)\right)+\frac{\partial \beta_{2}}{\partial z_{2}^{\prime}} \dot{z}_{2}^{\prime}+\frac{\partial \beta_{2}}{\partial \xi_{01}} \dot{\xi}_{01}+\frac{\partial \beta_{2}}{\partial \xi_{02}} \dot{\xi}_{02} \\
& +\frac{\partial \beta_{2}}{\partial \xi_{12}} \dot{\xi}_{12}+\frac{\partial \beta_{2}}{\partial \hat{\vartheta}_{1}} \dot{\hat{\vartheta}}_{1}+\frac{\partial \beta_{2}}{\partial \hat{\vartheta}_{2}} \dot{\hat{\vartheta}}_{2}-c_{3}^{\prime} z_{3}^{\prime}-d_{3}\left(\frac{\partial \beta_{2}}{\partial z_{1}^{\prime}}\right)^{2} z_{3}^{\prime} \\
& -\psi_{3}(y)-k_{3}\left(y-\xi_{01}\right),
\end{aligned}
$$

where $d_{3}>0, d_{3} \in R, c_{3}^{\prime}>0, c_{3}^{\prime} \in R$, Eq. (66) will become

$\dot{z}_{3}^{\prime}=-c_{3}^{\prime} z_{3}^{\prime}-d_{3}\left(\frac{\partial \beta_{2}}{\partial z_{1}^{\prime}}\right)^{2} z_{3}^{\prime}-\frac{\partial \beta_{2}}{\partial z_{1}^{\prime}}\left(\xi_{12} \tilde{\vartheta}_{3}+\varepsilon_{2}\right)$

Then the complete CLF for AOBC design is selected as

$f_{3}=f_{2}+\frac{1}{2}\left(z_{3}^{\prime}\right)^{2}+\frac{1}{d_{3}} \varepsilon^{T} P_{0} \varepsilon+\frac{1}{2 \gamma_{3}^{\prime}} \tilde{\vartheta}_{3}^{2}$

Substituting (69) into the derivative of $f_{3}$ produces

$$
\begin{aligned}
\dot{f}_{3}= & \dot{f}_{2}-c_{3}^{\prime}\left(z_{3}^{\prime}\right)^{2}-\left(\sqrt{d_{3}} \frac{\partial \beta_{2}}{\partial z_{1}^{\prime}} z_{3}^{\prime}+\frac{\varepsilon_{2}}{2 \sqrt{d_{3}}}\right)^{2}+\frac{1}{4 d_{3}} \varepsilon_{2}^{2} \\
& -\frac{1}{d_{3}} \varepsilon^{T} \varepsilon-\tilde{\vartheta}_{3}\left(\frac{\partial \beta_{2}}{\partial z_{1}^{\prime}} z_{3}^{\prime} \xi_{12}+\frac{1}{\gamma_{3}^{\prime}} \dot{\hat{\vartheta}}_{3}\right)
\end{aligned}
$$

where

$\frac{1}{4 d_{3}} \varepsilon_{2}^{2}-\frac{1}{d_{3}} \varepsilon^{T} \varepsilon=-\frac{1}{d_{3}} \varepsilon_{1}^{2}-\frac{3}{4 d_{3}} \varepsilon_{2}^{2}-\frac{1}{d_{3}} \varepsilon_{3}^{2}$. 


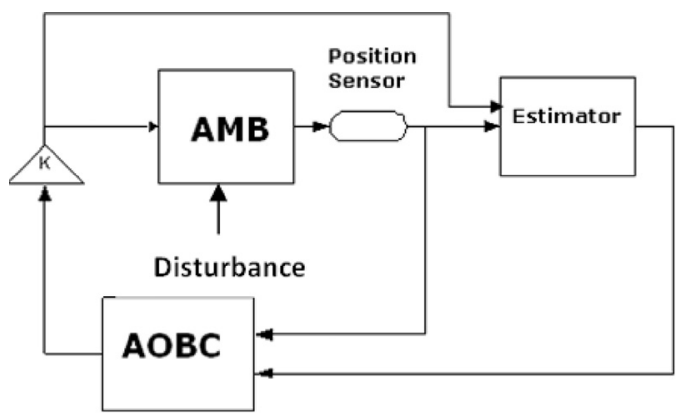

Fig. 5. AOBC controlled AMB system.
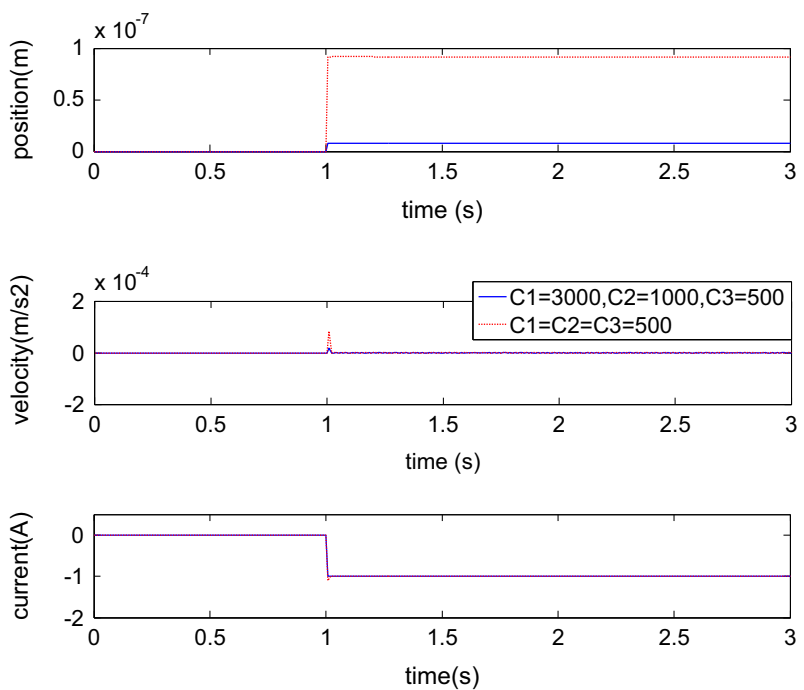

Fig. 6. Time responses of three states with different LCs.

If we choose adaptive law as

$\dot{\hat{\vartheta}}_{3}=-\gamma_{3}^{\prime} \frac{\partial \beta_{2}}{\partial z_{1}^{\prime}} z_{3}^{\prime} \xi_{12}$,

Eq. (71) will be negative semi definite. According to Lyapunov's direct method, the AOBC controlled AMB system is stable around equilibrium points. Then we can use Barbalat's Lemma to prove that the estimation errors of observer and disturbance are converging to zero as time goes to infinity. The Barbalat's Lemma is given as follows.

Barbalat's Lemma. [24]: If the differentiable function $\varphi(t)$ has a finite limit as $t \rightarrow \infty$, and if $\dot{\varphi}(t)$ is uniformly continuous, then $\dot{\varphi}(t) \rightarrow 0$ as $t \rightarrow \infty$.

To apply Barbalat's lemma to the analysis of dynamic systems, one typically uses the following immediate corollary, which looks very much like an invariant set theorem in Lyapunov analysis for time-invariant systems.

Lyapunov-Like Lemma. [24]: If a Lyapunov function $f$ satisfies the following conditions

- $f$ is lower bounded:

- $\dot{f}$ is negative semi-definite;

- $\dot{f}$ is uniformly continuous in time;

Then $\dot{f} \rightarrow 0$ as $t \rightarrow \infty$.

We know $f_{3}$ is lower bounded since $f_{3} \geq 0$. Eq. (71) shows $\dot{f}_{3}$ is negative semi definite and continuous. Therefore, from LyapunovLike Lemma, $\dot{f}_{3}$ goes to zero as time goes to infinite. Then

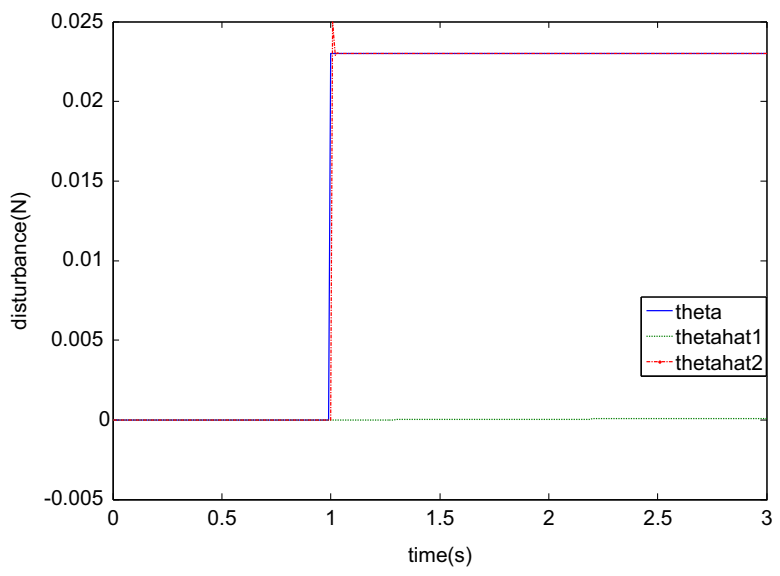

Fig. 7. Disturbance $(\theta)$ and its estimates when $c_{1}=c_{2}=c_{3}=500$.

$\varepsilon_{1}, \varepsilon_{2}, \varepsilon_{3}, \tilde{\vartheta}_{1}, \tilde{\vartheta}_{2}$ and $\tilde{\vartheta}_{3}$ are converging to zero as $t \rightarrow \infty$. Fig. 5 shows closed-loop diagram of an AOBC controlled AMB system where position output is the only feedback signal from AMB to controller. The estimator in Fig. 5 is used to estimate both disturbance and system's states. The AOBC is constructed based on the estimated states and disturbance.

\section{Simulation results}

We construct the $\mathrm{ABC}$ and $\mathrm{AOBC}$ controlled $\mathrm{AMB}$ systems respectively in Matlab/Simulink. The Simulink models for both control systems are based on Figs. 4 and 5. An external disturbance is added to the system as a step input as $t=1 \mathrm{~s}$. We use the parameter values for AMB given in Table 1. The nominal air gap is $0.7 \mathrm{~mm}$. All the initial values of state variables are assumed to be zeros.

\subsection{Tracking performance and disturbance estimation}

\subsection{1. $A B C$ controlled $A M B$ system}

As indicated in Section 3, for an ABC controlled AMB system, the real magnitude of an external disturbance is calculated as $\theta=\left(F_{s} / b m\right)=0.023$. In the following part, two sets of simulation results are given with different Lyapunov back stepping coefficients (LCs) $c_{i}(i=1,2,3)$ and adaptive coefficients (ACs) $\gamma_{i}(i=1,2)$ respectively for the purpose of investigating how these coefficients affect the control results of three states and disturbance estimate.

Fig. 6 shows the time responses of the three states $(x, v$, and $i)$ for different LCs as AC values are $\gamma_{1}=1$, and $\gamma_{2}=1$. Fig. 7 shows the disturbance estimation as LC values are $c_{1}=c_{2}=c_{3}=500$. Fig. 8 shows the disturbance estimation as LC values are chosen as $c_{1}=3000, c_{2}=1000, c_{3}=500$. In both Figs. 7 and 8, AC values are unchanged $\left(\gamma_{1}=1\right.$, and $\left.\gamma_{2}=1\right)$. From Fig. 6 , it is observed that the peak value of rotor's displacement is driven to less than $1 \times 10^{-7} \mathrm{~m}$ that can guarantee the rotor not touching stator. From Figs. 7 and 8 , we can see that by increasing LC values without changing ACs, the overshoot of the displacement could be remarkably reduced.

In order to investigate the effects of AC, we change their values in the following simulation while leaving $\mathrm{LC}$ values unchanged. We choose $c_{1}=3000, c_{2}=1000, c_{3}=500$. We increase the first $\mathrm{AC} \gamma_{1}$ from 1 to 3000, and decrease the second $A C \gamma_{2}$ from 1 to 0.1. The simulation results for different ACs are shown in Figs. 9-11. Fig. 9 shows the time responses of three states for different ACs. The peak value of rotor's displacement is driven to less than $1 \times 10^{-8} \mathrm{~m}$. Velocity and current are stabilized at their steady 


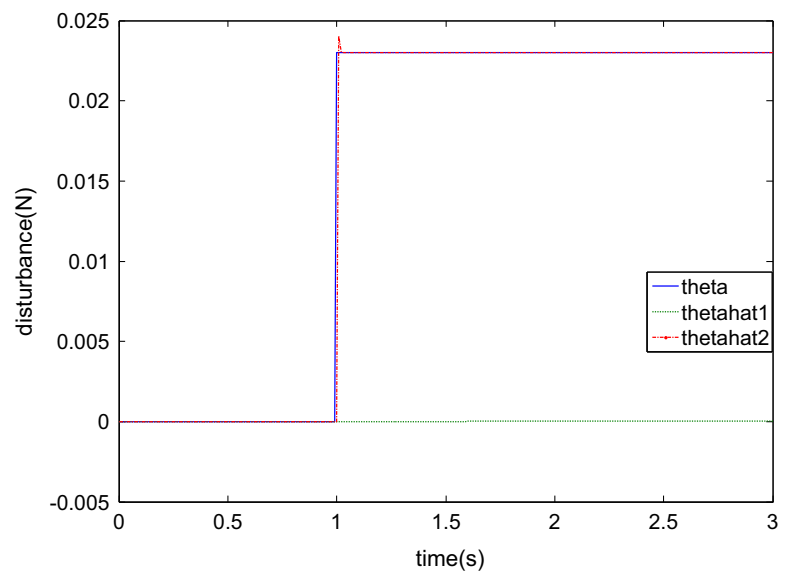

Fig. 8. Disturbance $(\theta)$ and its estimates when $c_{1}=3000, c_{2}=1000, c_{3}=500$.
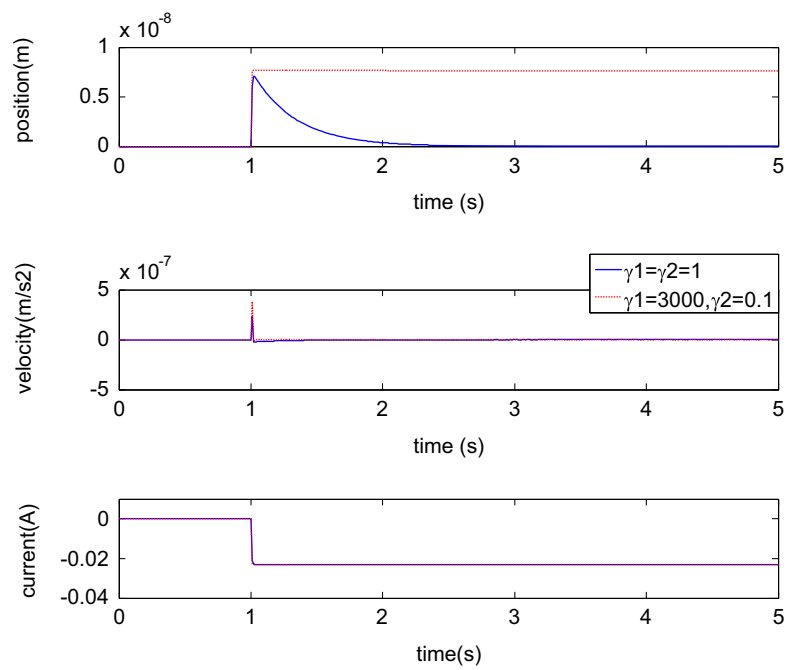

Fig. 9. Time responses of three states with different AC.

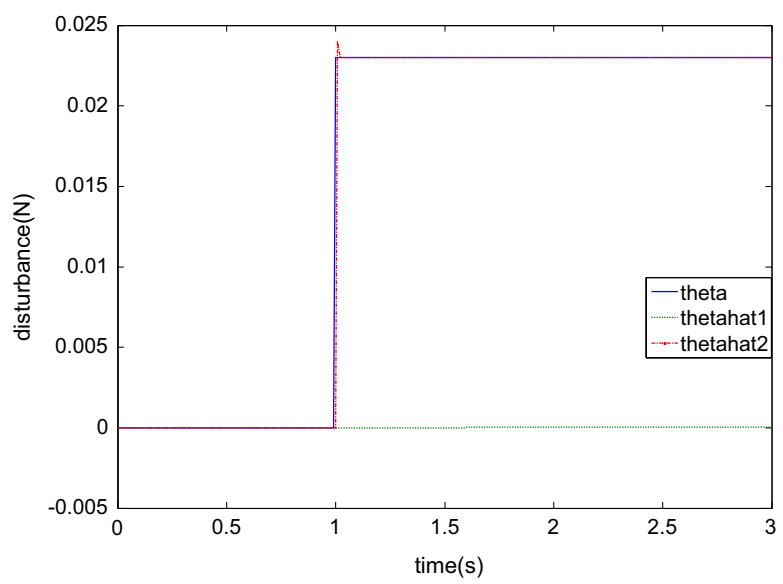

Fig. 10. Disturbance $(\theta)$ and its estimates when $\gamma_{1}=10$, and $\gamma_{2}=1$.

state values. Both Figs. 10 and 11 show that the external disturbance $\theta$ is successfully estimated by $\hat{\theta}_{2}$, which is represented by red colored dotted line. The settling time for estimated disturbance relies on the values of ACs. Increasing AC values can increase the estimation speed of external disturbance.

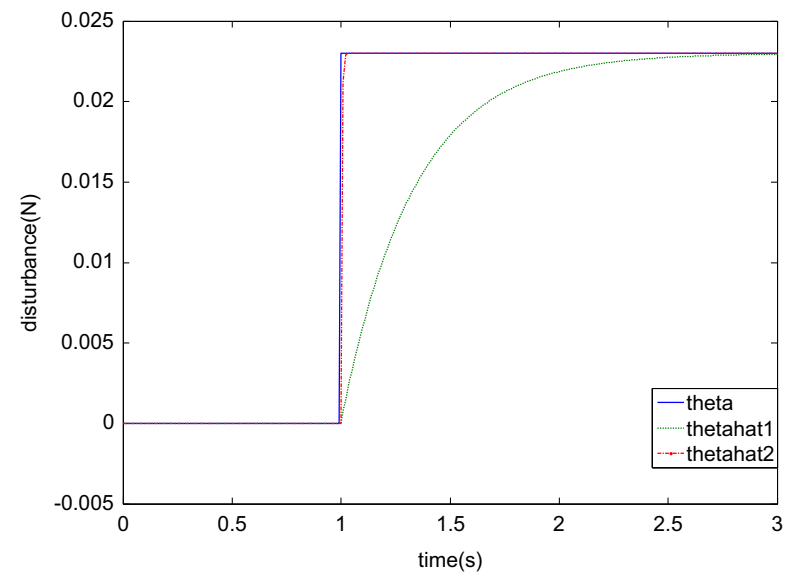

Fig. 11. Disturbance $(\theta)$ and its estimates when $\gamma_{1}=2000$, and $\gamma_{2}=0.1$.

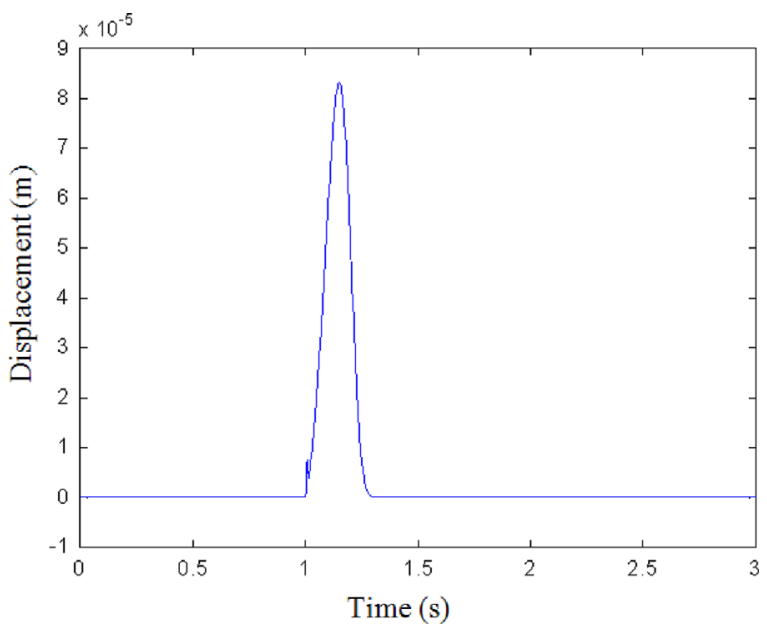

Fig. 12. Position output for an $A O B C$ controlled AMB.

As shown from Figs. 6 through 11, the displacement of the rotor in $\mathrm{AMB}$ has been successfully controlled to almost zero without steady state error by the ABC with different LCs and ACs. The adaptive laws estimate disturbance precisely. In addition, the LCs play an important role in system's response. The larger the LCs values are, the smaller the overshoot values will be. Increasing ACs can amplify the adaptation signals. Consequently the settling time for the estimation errors of disturbance is reduced.

\subsection{2. $A O B C$ controlled $A M B$ system}

For an AOBC controlled AMB system, the real magnitude of an external disturbance is calculated as $\vartheta=F_{d} / m=1$. The disturbance is added to the system at $t=1 \mathrm{~s}$ as a step input. The $\mathrm{LC}$ values are chosen as $c_{1}^{\prime}=5000, \quad c_{2}^{\prime}=1000, c_{3}^{\prime}=50$. The $A C$ values are chosen as $\gamma_{1}^{\prime}=15,000, \gamma_{2}^{\prime}=100, \gamma_{3}^{\prime}=1$. The coefficients $d_{i}$ are chosen as $d_{1}=d_{2}=d_{3}=1 \times 10^{-5}$. Fig. 12 shows the displacement output of the rotor under the control of $A O B C$. From the figure, we can see that the peak value of the displacement is controlled to be $8 \times 10^{-5} \mathrm{~m}$, which is much less than the nominal air gap $(0.7 \mathrm{~mm})$ in the presence of disturbance. The displacement is eventually stabilized at almost zero.

Fig. 13 shows the estimated disturbances $\left(\hat{\vartheta}_{1}, \hat{\vartheta}_{2}, \hat{\vartheta}_{3}\right)$, which are represented by blue, green, and red lines respectively, through three adaptive laws of AOBC. It is demonstrated that the disturbance is precisely estimated by the adaptive laws. 


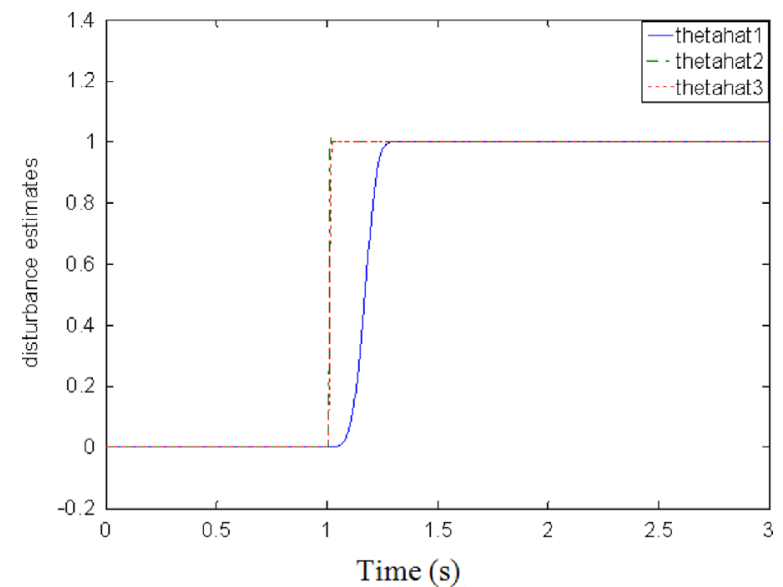

Fig. 13. Disturbance estimates by three adaptive laws. (For interpretation of the references to color in this figure, the reader is referred to the web version of this article.)

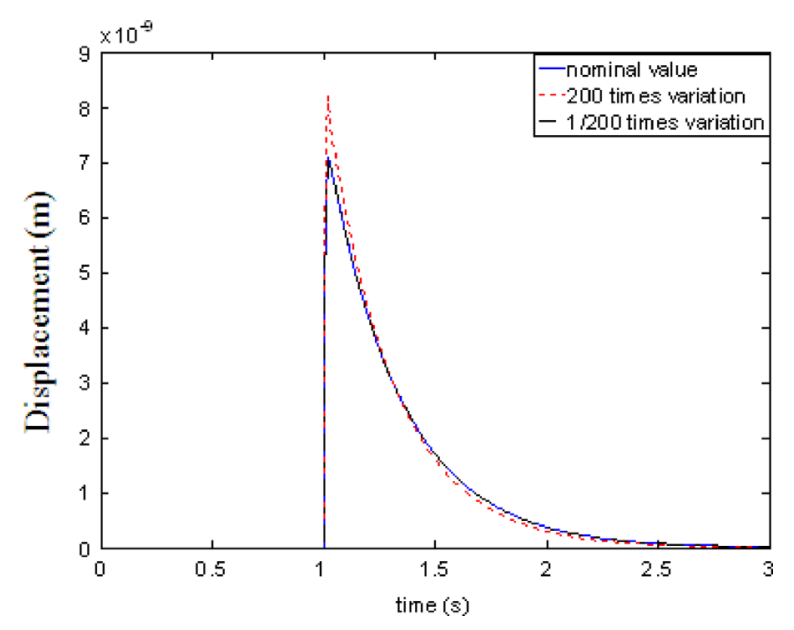

Fig. 14. Displacement output of $\mathrm{ABC}$ controlled $\mathrm{AMB}$ with variant $a$.

\subsection{Robustness against parameter variations}

\subsection{1. $A B C$ controlled $A M B$ system}

$A B C$ 's robustness is tested by changing the system's parameter $a$, which is defined in (17), by 200 and $1 / 200$ times from their original values. Fig. 14 shows rotor's displacement with a variant parameter $a$. A step disturbance is added to the system at $t=1 \mathrm{~s}$. The figure shows the convergence of the displacement to zero in the presence of parameter variations and external disturbance. When we change other parameters $b, c, d$ in (17) by 200 and $1 / 200$ times from their original values, we obtain the same simulation result as in Fig. 14.

\subsection{2. $A O B C$ controlled $A M B$ system}

We keep the LC and AC values unchanged. A step disturbance is added to the system at $1 \mathrm{~s}$. We vary the parameter $a^{\prime}$ in (43) from $(1 / 2.3) a^{\prime}$ to $2.3 a^{\prime}$ without tuning the observer parameters. Fig. 15 shows the displacement of the rotor with the variance of $a^{\prime}$. From the figure, we can see that the peak value of the displacement is less than nominal air gap $(0.7 \mathrm{~mm})$. When we change the parameters $b^{\prime}$ and $c^{\prime}$ from their nominal values to 2.3 times their nominal values, we obtain the same displacement output as shown in Fig. 15. However, if we increase $a^{\prime}, b^{\prime}$ and $c^{\prime}$ by over 2.3 times, the peak value of the displacement will exceed nominal air gap, causing the system unstable. Comparing Figs. 14 and 15,

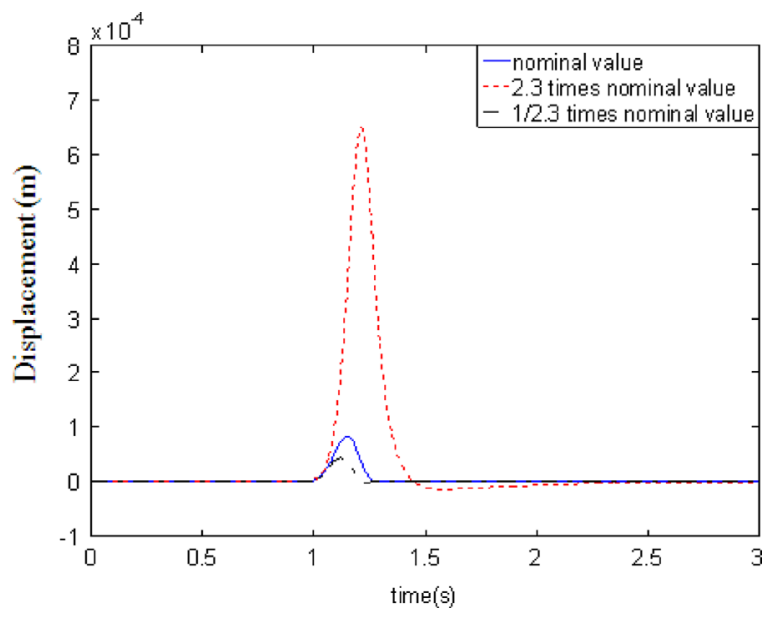

Fig. 15. Displacement output of AOBC controlled AMB with variant $a^{\prime}$.

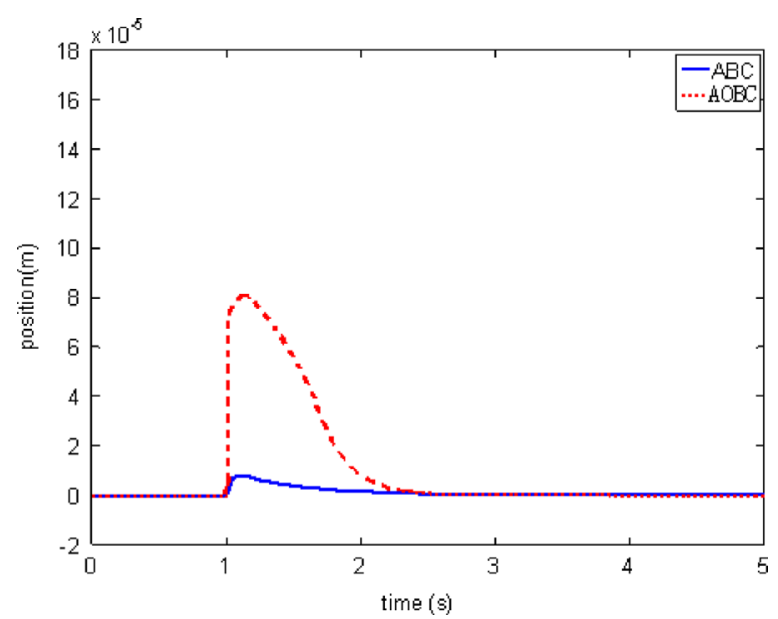

Fig. 16. Position responses of $A O B C$ and $A B C$ controlled $A M B$ systems.

we can see that the displacement output of $A O B C$ controlled AMB has much larger overshoot value than that of $A B C$ controlled $A M B$.

\subsection{Comparison between $A B C$ and $A O B C$}

From simulation results (Figs. 6 through 15), we can see that both $\mathrm{ABC}$ and $\mathrm{AOBC}$ successfully control the rotor's displacement within the nominal air gap in an $A M B$ system despite the presences of disturbance and parameter variations. In addition, the external disturbance is precisely estimated by the adaptive laws of $A B C$ and $A O B C$ respectively. However, the $A B C$ demonstrates better robustness than $\mathrm{AOBC}$ against disturbance and parameter variations. Fig. 16 shows the displacement outputs for $\mathrm{ABC}$ and $\mathrm{AOBC}$ controlled $\mathrm{AMB}$ systems after a step disturbance is added to the system at $t=1 \mathrm{~s}$. As shown in Fig. 16, the displacement output of an $\mathrm{AOBC}$ controlled $\mathrm{AMB}$ has larger overshoot value than that of $\mathrm{ABC}$ controlled $\mathrm{AMB}$. Comparing Fig. 14 with Fig. 15, we can see that when we vary system parameters, AOBC controlled AMB has larger overshoot in displacement output than $\mathrm{ABC}$ too. This is because the controller in $\mathrm{AOBC}$ is based on the observed states of an observer which has the observation errors decreasing with time. In addition, AOBC has more tuning parameters than $\mathrm{ABC}$. There are six controller parameters $\left(c_{1}^{\prime}, c_{2}^{\prime}, c_{3}^{\prime}, d_{1}, d_{2}, d_{3}\right)$, three adaptive parameters $\left(\gamma_{1}^{\prime}, \gamma_{2}^{\prime}, \gamma_{3}^{\prime}\right)$, and three observer parameters $\left(k_{1}, k_{2}, k_{3}\right)$ to tune for an AOBC while there are only three controller $\left(c_{1}, c_{2}, c_{3}\right)$ and three adaptive parameters $\left(\gamma_{1}, \gamma_{2}, \gamma_{3}\right)$ in an ABC. A large number of tuning 


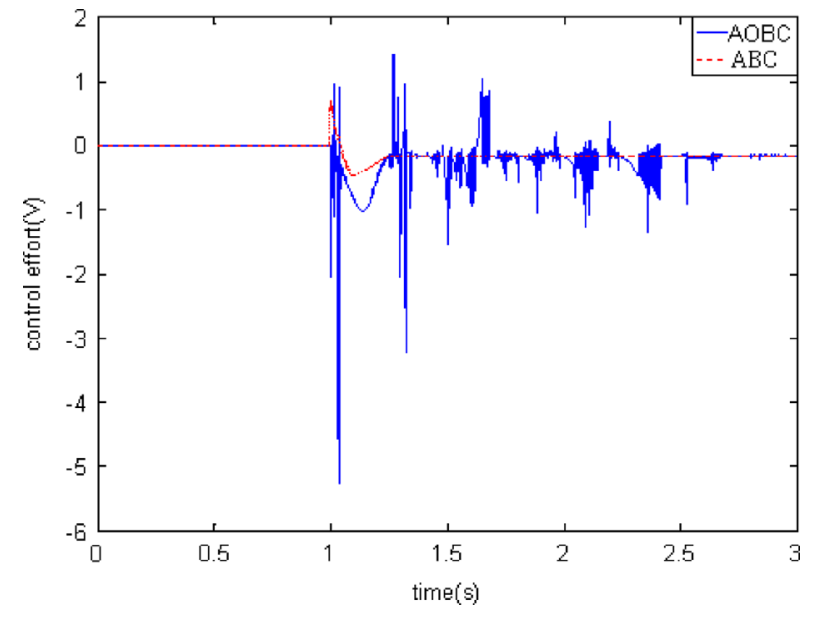

Fig. 17. Control efforts of $A B C$ and $A O B C$.

parameters makes the $\mathrm{AOBC}$ more difficult to implement than $\mathrm{ABC}$ in the real world. Fig. 17 shows the control efforts for both $A B C$ and $A O B C$. The control effort of $A B C$ is smooth while the one for $A O B C$ is oscillatory. Nevertheless, $\mathrm{AOBC}$ only needs one available state from $A M B$ (rotor's displacement) while $A B C$ requires full state outputs (displacement, velocity, and current) from AMB. This advantage makes $A O B C$ is a better control option than $A B C$ for a real $A M B$ system where current and velocity are not measurable. Fig. 16 shows the position outputs for $A B C$ and $A O B C$ controlled AMB systems.

\section{Concluding remarks}

Two types of adaptive control methods, adaptive back stepping control $(\mathrm{ABC})$ and adaptive observer based back stepping control $(A O B C)$ are applied to an active magnetic bearing (AMB) system. The $A B C$ is based on full state feedback (displacement, velocity, and current) from the $A M B$ while the $A O B C$ is constructed on a single feedback signal (displacement). Both $A B C$ and $A O B C$ achieve excellent control performances in regulating a rotor's position in the $A M B$, in disturbance rejection, and in being robust against system uncertainties. Lyapunov's direct method proves the stability of two control systems under the interference of disturbance. Simulation results verify the effectiveness and robustness of both control systems. The $\mathrm{ABC}$ and $\mathrm{AOBC}$ methods introduced in the paper can also be applied to the other nonlinear system models from which a linearized "strict feedback form" and observable canonical form can be obtained.

Adaptive back stepping control has the potential to be widely applied in the real world with its reliability and the ability of online estimation of uncertainties. However, the tuning of multiple parameters for controller and adaptive laws makes it difficult to implement in practice. In the future, a systematic tuning method of the controller parameters needs to be discovered. A study about how to accurately choose Lyapunov and adaptive coefficients will be conducted since their variations influence the system's performance. We also plan to implement the $A O B C$ on a real $A M B$ of the flywheel energy storage system in NASA Glenn Research Center at Cleveland, $\mathrm{OH}$.

\section{References}

[1] Mukhopadhyay SC, Gooneratne C, Sen Gupta G. Magnetic bearing: an integrated platform for teaching and learning. In: Proceedings of the 2nd international conference on autonomous robots and agents. Palmerston North, New Zealand; December 2004. p. 283-8.

[2] Rarick Richard A. Control of an active magnetic beating with and without position sensing (Master's thesis). Cleveland, OH: Department of Electrical and Computer Engineering, Cleveland State University; 2007.

[3] Polajžer B, Ritonja J, Štumberger G, Dolinar D, Lecointe JP. Decentralized PI/PD position control for active magnetic bearings. Electr Eng 2006;89(1):53-9.

[4] Arredondo I, Jugo J, Etxebarria V. Modeling and control of a flexible rotor system with AMB based sustentation. ISA Trans 2008;47(1):101-12.

[5] Denver Timothy P, Brown Gerald V, Jansen Ralph H. Estimator based controller for high speed flywheel magnetic bearing system. NASA internal report (NASA/TM-2002-211795); August 2002.

[6] Kim Ha-Yong, Lee Chong-Won. Design and control of active magnetic bearing system with Lorentz force type axial actuator. Mechatronics 2006;16 (1):13-20.

[7] Jastrzebski Rafal Piotr, Pollanen Riku. Centralized optimal position control for active magnetic bearings: comparison with decentralized control. Electr Eng 2009;91(2):101-14.

[8] Grega Wojciech, Pilat Adam. Comparison of linear control methods for an AMB system. Int J Appl Math Comput Sci 2005;15(2):245-55.

[9] Kucera Ladislav. Robustness of self sensing magnetic bearing. In: Proceedings of the magnetic bearings industrial conference. Alexandria, Virginia; 1991. p. 261-70.

[10] Li Lichuan, Shinshi Tadahiko, Shimokohbe Akira. State feedback control for active magnetic bearing based on current change rate alon". IEEE Trans Magn 2004;40(6):3512-7.

[11] Noh Myounggyu D, Maslen Eric H. Self sensing magnetic bearings using parameter estimation. IEEE Trans Instrum Meas 1997;46(1):45-50.

[12] Su-Alexander B, Rarick R, Dong L. A novel application of an extended state observer for high performance control of NASA's HSS flywheel. In: Proceedings of 2008 American control conference. Seattle, WA; June 11-13, 2008. p. 5216-21.

[13] Kristic Miroslav, Kanellakopoulos Ioannis, Kokotavic Petar. Nonlinear and adaptive control design. New York: Wiley-Interscience; 1995.

[14] Lu CH. Hwang YR, Shen YT. Backstepping sliding mode tracking control of a vane-type air motor $X-Y$ table motion system. ISA Trans 2011;50(2):278-86.

[15] Jiang Ye, Hu Qinglei, Ma Guangfu. Adaptive backstepping fault-tolerant control for flexible spacecraft with unknown bounded disturbances and actuator failures. ISA Trans 2010;49(1):57-69.

[16] Adhikary Nabanita, Mahanta Chitralekha. Integral backstepping sliding mode control for underactuated systems: Swing-up and stabilization of the CartPendulum System. ISA Trans 2013;52(6):870-80.

[17] Yang Jung-Hua, Hsu Wen-Chun. Adaptive backstepping control for electrically driven unmanned helicopter. Control Eng Pract 2009;17(8):903-13.

[18] Joshi RR, Gupta RA, Wadhwani AK. Adaptive backstepping controller design and implementation for a matrix converter based IM drive system. J Theor Appl Inf Technol 2007;3(2):28-41.

[19] Boussehane IK, Hazzad A, Rahli M, Mazari B, Kamli M. Mover position control of linear induction motor drive using adaptive backstepping controller with integral action. J Sci Eng 2009;12(1):17-28.

[20] Farivar F, Shoorehdeli MA, Nekoui MA, Teshnehlab M. Gaussian radial basis adaptive backstepping control for a class of nonlinear system. J Appl Sci 2009;9(2):248-57.

[21] Sivrioglu Selim, Nonami Kenzo. Adaptive output backstepping control of a flywheel zero-bias AMB system with parameter uncertainty. Nonlinear Dyn 2007; $48(1-2): 157-84$.

[22] Kokotovic PV. Joy of feedback: nonlinear and adaptive. Control Syst Mag 1992;12(3):7-17.

[23] Dong L, You S. Adaptive backstepping control of active magnetic bearings. In: Proceedings of the IEEE conference on control and automation. Hangzhou, China; June 12-14, 2013. p. 452-7.

[24] Slotine J, Li W. Applied nonlinear control. NJ: Prantice-Hall, Englewood Cliffs; 1991. 